\title{
ELECTRON-POSITRON ANNIHILATION INTO HADRONS
}

\section{Robert N. Cahn and John Ellis}

\author{
Erratum and Addendum
}

Table III of this paper was inadvertently omitted. It should have appeared after its first mention at the bottom of $p_{0} 6$ 。 It would have shown four quarks: $p, n, \lambda$ and $\mathrm{p}^{\prime}$ $\left(\mathrm{Q}=2 / 3, \mathrm{I}_{\mathrm{Z}}=0, \mathrm{Y}=-2 / 3\right.$, Charm $\left.=1\right)$. Table IV on p. 7 has been mislabeled Table III. Table IV should appear on the previous page, preceding the sentence "This scheme is rather complicated:..." 


\title{
ELECTRON-POSITRON ANNIHILATION INTO HADRONS*
}

\author{
Robert N. Cahn† \\ Stanford Linear Accelerator Center \\ Stanford University, Stanford, Calif. 94305 \\ John Ellis †† \\ California Institute of Technology, Pasadena, Calif.
}

\section{ABSTRACT}

Theories about the behavior of the ratio

$$
\frac{\sigma\left(\mathrm{e}^{+} \mathrm{e}^{-} \rightarrow \gamma \rightarrow \text { hadrons }\right)}{\sigma\left(\mathrm{e}^{+} \mathrm{e}^{-} \rightarrow \gamma \rightarrow \mu^{+} \mu^{-}\right)}
$$

are reviewed, and qualitative features of the final state hadron distributions are discussed.

\section{INTRODUCTION}

The process $\mathrm{e}^{+} \mathrm{e}^{-} \rightarrow \gamma \rightarrow$ hadrons has long been a favorite playground of theoreticians. It is interesting because all theories which exhibit Bjorken scaling in electroproduction also make the naive scale invariant prediction ${ }^{1}$

$$
\sigma\left(\mathrm{e}^{+} \mathrm{e}^{-} \rightarrow \gamma \rightarrow \text { hadrons }\right) \underset{\mathrm{q}^{2} \rightarrow \infty}{\frac{1}{\mathrm{q}^{2}}}
$$

Hence if the prediction (1), or the equivalent (assuming QED) statement

$$
\mathrm{R}\left(\mathrm{q}^{2}\right) \equiv \frac{\sigma\left(\mathrm{e}^{+} \mathrm{e}^{-} \rightarrow \gamma \rightarrow \text { hadrons }\right)}{\sigma\left(\mathrm{e}^{+} \mathrm{e}^{-} \rightarrow \gamma \rightarrow \mu^{+} \mu^{-}\right)} \underset{\mathrm{q}^{2} \rightarrow \infty}{\longrightarrow} \mathrm{R}
$$

where $\mathrm{R}$ is some model-dependent constant, should fail, then we would have to conclude that we really do not understand scaling in electroproduction at all.

Experimental data from $\mathrm{CEA}^{2}$ seem to show little evidence that the asymptotic prediction (2) is being approached for $q^{2} \leq 25 \mathrm{GeV}$. In fact the hadron-muon ratio seems to be increasing, and is alrcady large compared with many theoretical predictions. (Though this trend may not be statistically significant at the present level of accuracy of the data.) Hence it is very important:

\footnotetext{
*Work supported in part by the U. S. Atomic Energy Commission.

$\dagger$ Present address: Physics Department, Univ. of Washington, Seattle 98195.

†† Present address: Theory Division, CERN, 1211 Geneva 23, Switzerland.
}

(Presented at the 1973 Meeting of the Division of Particles and Fields of the A PS, Berkeley, California, August 13-17, 1973) 
1. To confirm the CEA experiments.

2. To see whether $R\left(q^{2}\right) \rightarrow \infty$ as $q^{2} \rightarrow \infty$ could be accommodated theoretically.

3. To try to understand an increase in $R$ over a range in $q^{2}$.

4. To understand implications elsewhere, e.g. , for $Q E D$, of $R\left(q^{2}\right)$ being large, and

5. To see what theoretical values for $R$ are still plausible. We leave 1. to SPEAR, and here review 2. to 5. Data either are or will shortly be available from CEA and SPEAR on topological cross section, the multiplicity and higher moments of the multiplicity distribution, and we discuss these qualitative features of the hadronic final state. Detailed data on the inclusive distributions and their scaling behavior are rather further away and we do not discuss them in detail here: there is more about them in the parallel session on partons and the light-cone.

\section{THE ASYMPTOTIC BEHAVIOR OF R( $\left.q^{2}\right)$}

Since it has the dimensions of (length $)^{2}, \sigma\left(\mathrm{e}^{+} \mathrm{e}^{-} \rightarrow \gamma \rightarrow\right.$ hadrons) would have to go as $1 / q^{2}$ as $q^{2} \rightarrow \infty$ in order to be scale invariant, and this is what is predicted in simple parton ${ }^{3}$ and canonical short distance behavior 4 models of scaling. Since according to $\operatorname{QED}\left(\mathrm{e}^{+} \mathrm{e}^{-} \rightarrow \gamma \rightarrow \mu^{+} \mu\right\urcorner \sim 1 / \mathrm{q}^{2}$ also, this would mean that $R\left(q^{2}\right)$ approaches a constant $R$ as $q^{2} \rightarrow \infty$. Setting aside oscillations, the other possibilities are that $R\left(q^{2}\right) \rightarrow 0$ or $\infty$. The former of these is not suggested by the data and is unfashionable theoretically. If $R\left(q^{2}\right) \rightarrow \infty$, might go as a power of $\ln q^{2}$ or as a power of $q^{2}$ itself. There seems to be nothing wrong in principle with $R\left(q^{2}\right)-(\ln q)^{n}$ for some $n>0$ : indeed this is what occurs in any finite order of a renormalizable field theory (except for gauge theories, 5,6 of which more later). Indeed one actually expects that the naive scaling prediction (1) will be modified by terms $0\left(\alpha \ln \mathrm{q}^{2} / \mathrm{q}^{2}\right)$ etc., when radiative corrections to the strong interactions are taken into account (cf. electroproduction) 7 .

Could $R\left(q^{2}\right) \sim\left(q^{2}\right)^{m}$ for some power $m>0$ as $q^{2} \rightarrow \infty$ ? We know of no theory where this occurs, and it can be argued that such a possibility would destroy the renormalizability of QED. Consider for example the contribution of the high $\mathrm{q}^{2}$ part of the hadron vacuum polarization to the muon $(\mathrm{g}-2)$ shown in Fig. 1. It is given by

$$
\Delta(\mathrm{g}-2)_{\mu}=\frac{\mathrm{m}_{\mu}^{2}}{6 \pi^{3}} \int_{\mathrm{Q}^{2}}^{\infty} \frac{\sigma\left(\mathrm{e}^{+} \mathrm{e}^{-} \rightarrow \gamma \rightarrow \text { hadrons }\right)}{\mathrm{q}^{2}} \mathrm{dq} \mathrm{q}^{2}
$$

where $\mathrm{Q}^{2}$ is some lower limit $>\mathrm{m}_{\mu}^{2}$. It is clear that the integral diverges if $\left(\mathrm{e}^{+} \mathrm{e}^{-} \rightarrow \gamma \rightarrow\right.$ hadrons) goes to a constant as $\mathrm{q}^{2} \rightarrow \infty$, entailing a subtraction and a new renormalization constant. Indeed, simple Dyson power counting indicates that an infinite number of QED graphs become divergent if $\left(\mathrm{e}^{+} \mathrm{e}^{-} \rightarrow \gamma \rightarrow\right.$ hadrons $) \sim\left(\mathrm{q}^{2}\right)^{\mathrm{m}}$ for any $\mathrm{m}>0$, because internal photon propagators acquire $\alpha\left(q^{2}\right)^{\mathrm{m}-1}$ corrections. Actually, in such theories even an expansion in powers of $\alpha$ would be invalid: we can only use a photon 
propagator (see Fig. 2)

$$
P\left(q^{2}\right)=\frac{1}{q^{2}}\left(1+\Pi\left(q^{2}\right)\right)
$$

where

$$
\frac{\Pi\left(q^{2}\right)}{q^{2}}=\frac{1}{4 \pi^{2} \alpha} \int \mathrm{ds} \frac{\sigma \mathrm{e}^{+} \mathrm{e}^{-} \rightarrow \gamma \rightarrow \text { hadrons }}{(\mathrm{s})}
$$

when

$$
P\left(q^{2}\right)=\frac{1}{q^{2}} \frac{1}{1-\Pi\left(q^{2}\right)}
$$

as in Fig. 3. Clearly one expects that the rise in $R\left(q^{2}\right)$ should cut off when $\left|\Pi\left(q^{2}\right)\right|<1$ : for $R\left(q^{2}\right) \sim\left(q^{2}\right)^{1 / 2}, \Pi \sim 0.1$ when $\left(q^{2}\right)^{1 / 2} \sim 35 \mathrm{GeV}$.

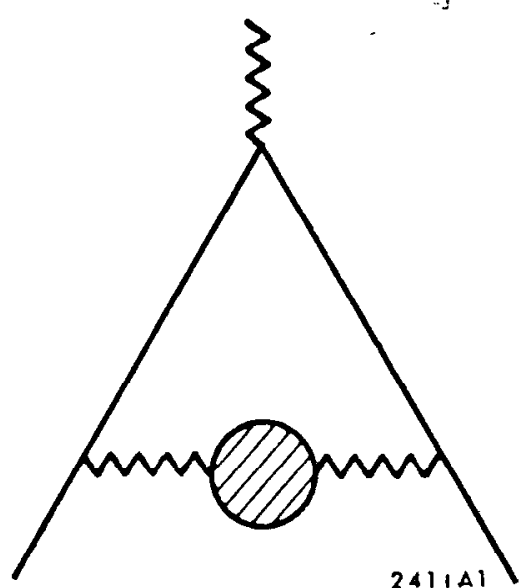

FIG. 1--Hadronic contribution to the vacuum polarization corto $\mathrm{g}-2$ of the muon.

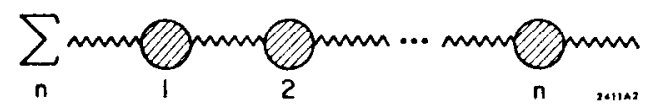

FIG. 2--Geometric series responsible for the full vacuum polarization correction to the photon propagator.

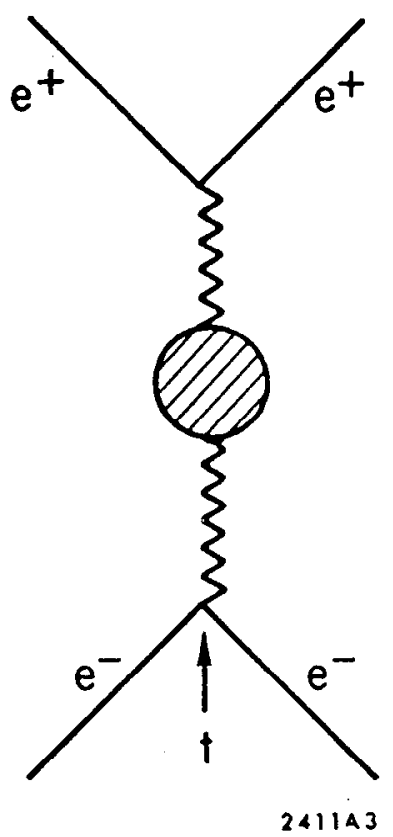

FIG. 3--Hadronic vacuum polarization correction for $t$-channel photon exchange in $\mathrm{e}^{+} \mathrm{e}^{-}$elastic scattering.

One major piece of recent theoretical progress has been the realization $^{5,6}$ that some renormalizable field theories have the naive scaling law (1). These are a class of non-Abelian gauge theories (i.e., theories with 
a non-commutative symmetry group in which a set of vector mesons are present whose transformation properties are such that the theory is invariant under "rotations" within the group even when the "rotation" is a function of $x$, the space-time point), which have for some time been popular among constructors ${ }^{8}$ of renormalizable theories of weak interactions, and those trying to understand how quarks might be bound in hadrons. ${ }^{9}$ It has been shown that these theories are "asymptotically free" which means that at short distances and near the light cone their singularity structures are almost the same as those of a free field theory. Bjorken scaling in electroproduction in such theories is violated by calculable inverse powers of ln $q^{2} .10$ The naive parton or canonical short distance model scaling prediction

$$
\frac{\sigma\left(\mathrm{e}^{+} \mathrm{e}^{-} \rightarrow \gamma \rightarrow \text { hadrons }\right)}{\sigma\left(\mathrm{e}^{+} \mathrm{e}^{-} \rightarrow \gamma \rightarrow \mu^{+} \mu^{-}\right)} \rightarrow \mathrm{R}
$$

is valid in these models. Also, sub-asymptotic inverse logarithm corrections to (5) can be calculated as discussed later.

\section{WHAT IS R?}

Let us now assume that $R\left(q^{2}\right)$ approaches a constant, $R$, as $q^{2} \rightarrow \infty$ in accord with theoretical prejudice. In all theoretical models $3,4,5$ this occurs because at large $\mathrm{q}^{2}$ the electromagnetic current acts as if it is coupled to point-like spin-1/2 fields (quarks) in addition to the usual leptons:

$$
\mathrm{J}_{\mu}^{\mathrm{e} . \mathrm{m} .} \propto-\overline{\mathrm{e}} \gamma_{\mu} \mathrm{e}-\bar{\mu} \gamma_{\mu} \mu+\sum_{\mathrm{i}} \mathrm{Q}_{\mathrm{i}} \overline{\mathrm{q}}_{\mathrm{i}} \gamma_{\mu} \mathrm{q}_{\mathrm{i}}
$$

where the sum is over all quarks, $q_{i}$, with charges $Q_{i}$. Since each quark enters analogously to a muon,

$$
\mathrm{R}\left(\mathrm{q}^{2}\right) \equiv \frac{\sigma\left(\mathrm{e}^{+} \mathrm{e}^{-} \rightarrow \gamma \rightarrow \text { hadrons }\right)}{\sigma\left(\mathrm{e}^{+} \mathrm{e}^{-} \rightarrow \gamma \rightarrow \mu^{+} \mu^{-}\right)} \underset{\mathrm{q}^{2} \rightarrow \infty}{\longrightarrow} \sum_{\mathrm{i}} \mathrm{Q}_{\mathrm{i}}^{2}
$$

(Spin-0 quarks enter with a contribution $\mathrm{Q}_{\mathrm{i}}^{2} / 4$.) Thus for the usual GellMann-Zweig ${ }^{11}$ scheme $R=4 / 9+1 / 9+1 / 9=2 / 3$.

There are both experimental and theoretical reasons for considering alternative quark schemes. Early colliding beam results give $R\left(q^{2}\right)>1$ and perhaps $R\left(q^{2}\right)>2-3$. Theoretically, the $\pi^{0} \rightarrow 2 \gamma$ decay rate can be related to short distance behavior ${ }^{12}$ where we hope the electromagnetic current has the simple form indicated in (6). Together with PCAC, the Gell-MannZweig scheme gives a rate too small by a factor of about 10 .

Also the Gell-Mann-Zweig scheme has well-known intrinsic problems with statistics. One solution is to invoke a new underlying symmetry by supposing there are several "colors" of each kind of quark. 13 To get the $\pi^{0} \rightarrow 2 \gamma$ decay rate right, one needs three colors - red, blue and yellow, say - giving a factor nine in the square of the amplitude. With three colors we can put three quarks in a symmetric 10 representation of the 
usual $(p, n, \lambda) \mathrm{SU}(3)$ and in an anti-symmetric 1 representation of color $\mathrm{SU}(3)$, making an overall anti-symmetric state without any further machinations.

More recently, further theoretical support has been given to "color" schemes. Lipkin, 9 following an old proposal of Nambu, 14 has pointed out that "colored gluons" i.e., vector mesons belonging to an 8 representation of color SU(3) can reproduce the fact that only triality zero hadrons are observed. The result follows from a simplified treatment in which the quarks are very heavy but move non-relativistically with a lighter effective mass. Lipkin finds that only color singlets are bound while color $\underline{8}^{\prime} \mathbf{s}, \underline{3}$ 's etc., are unbound by energies of the order of the quark mass. Since all color singlets (e.g. , $\overline{R R}+\bar{B} B+\bar{Y} Y$ ) have triality zero, a fundamental fact is accounted for. Whether Lipkin's analysis can be used to study exoticity (i.e., the absence of $27^{\prime} \mathrm{s}$ of $\left.(\mathrm{p}, \mathrm{n}, \lambda) \mathrm{SU}(3)\right)$ is less clear, as is the question of whether his vector gluons are gauge vector gluons.

The rcalization 5 that non-Abelian gauge theories are asymptotically free has added impetus to the study of color schemes which furnish an underlying non-Abelian symmetry. Abelian gauge theories like the original GellMann-Zweig scheme with a neutral gauge vector gluon violate scaling by positive powers of $\ln \mathrm{q}^{2}$.

These recent theoretical developments have increased interest in color schemes which have been around for some time. We mention here a few such schemes in the hope that if $R\left(q^{2}\right)$ does approach a constant $q^{2} \rightarrow \infty$, then it will be an interpretable number $-2 / 3$ for example - rather than $\pi / 2$, say.

Table I Gell-Mainn-Zweig with Color

\begin{tabular}{cccc}
\hline & $\mathrm{Q}$ & $\mathrm{I}_{\mathrm{Z}}$ & $\mathrm{Y}$ \\
\hline $\mathrm{p}_{\mathrm{R}, \mathrm{Y}, \mathrm{B}}$ & $2 / 3$ & $1 / 2$ & $1 / 3$ \\
$\mathrm{n}_{\mathrm{R}, \mathrm{Y}, \mathrm{B}}$ & $-1 / 3$ & $-1 / 2$ & $1 / 3$ \\
$\lambda_{\mathrm{R}, \mathrm{Y}, \mathrm{B}}$ & $-1 / 3$ & 0 & $-2 / 3$ \\
\hline
\end{tabular}

$$
\begin{aligned}
J^{e m} & \alpha \frac{2}{3}\left(\bar{p}_{R} p_{R}+\bar{p}_{Y} p_{Y}+\bar{p}_{B} p_{B}\right)-\frac{1}{3}\left(\bar{n}_{R} n_{R}+\bar{n}_{Y} n_{Y}+\bar{n}_{B} n_{B}\right) \\
& -\frac{1}{3}\left(\bar{\lambda}_{R} \lambda_{R}+\bar{\lambda}_{Y} \lambda_{Y}+\bar{\lambda}_{B} \lambda_{B}\right)
\end{aligned}
$$

$\mathrm{J}^{\mathrm{em}}$ is a color singlet and a $(\mathrm{p}, \mathrm{n}, \lambda) \mathrm{SU}(3)$ octet, so that final states in $\mathrm{e}^{+} \mathrm{e}^{-}$annihilation are color singlets. $\mathrm{R}=3 \times 2 / 3=2$. 
Table II Han-Nambu 15

\begin{tabular}{cccccccc}
\hline & $\mathrm{Q}$ & $\mathrm{I}_{\mathrm{z}}$ & $\mathrm{Y}$ & & $\mathrm{Q}$ & $\mathrm{I}_{\mathrm{z}}$ & $\mathrm{Y}$ \\
\hline $\mathrm{p}_{\mathrm{R}}$ & 1 & $1 / 2$ & 1 & $\mathrm{p}_{\mathrm{B}}$ & 0 & 0 & 0 \\
$\mathrm{n}_{\mathrm{R}}$ & 0 & $-1 / 2$ & 1 & $\mathrm{n}_{\mathrm{B}}$ & -1 & -1 & 0 \\
$\lambda_{\mathrm{R}}$ & 0 & 0 & 0 & $\lambda_{\mathrm{B}}$ & -1 & $-1 / 2$ & -1 \\
$\mathrm{p}_{\mathrm{Y}}$ & 1 & 1 & 0 & & & \\
$\mathrm{n}_{\mathrm{Y}}$ & 0 & 0 & 0 & & & \\
$\lambda_{\mathrm{Y}}$ & 0 & $1 / 2$ & -1 & \\
\hline \hline \\
\hline
\end{tabular}

$\mathrm{J}^{\mathrm{em}}$ has singlet and octet pieces under both color and $(p, n, \lambda) \mathrm{SU}(3)$

groups. Hence the final states in $\mathrm{e}^{+} \mathrm{e}^{-}$annihilation include color octet pieces. If all final states are included, $R=4$. If only color singlets are permitted we must calculate

$$
\sum_{n \in 1}\langle 0|J| n\rangle\langle n|J| 0\rangle
$$

Inserting the contributing color singlet states

$$
\begin{aligned}
& \frac{1}{\sqrt{3}}\left(\overline{\mathrm{p}}_{\mathrm{R}} \mathrm{p}_{\mathrm{R}}+\overline{\mathrm{p}}_{\mathrm{Y}} \mathrm{p}_{\mathrm{Y}}+\overline{\mathrm{p}}_{\mathrm{B}} \mathrm{p}_{\mathrm{B}}\right), \\
& \frac{1}{\sqrt{3}}\left(\overline{\mathrm{n}}_{\mathrm{R}} \mathrm{n}_{\mathrm{R}}+\overline{\mathrm{n}}_{\mathrm{Y}} \mathrm{n}_{\mathrm{Y}}+\overline{\mathrm{n}}_{\mathrm{B}} \mathrm{n}_{\mathrm{B}}\right), \\
& \frac{1}{\sqrt{3}}\left(\bar{\lambda}_{\mathrm{R}} \lambda_{\mathrm{R}}+\bar{\lambda}_{\mathrm{Y}} \lambda_{\mathrm{Y}}+\bar{\lambda}_{\mathrm{B}} \lambda_{\mathrm{B}}\right),
\end{aligned}
$$

one finds that for these restricted final states, $R=2$.

Weak interaction theorists 16 have proposed introducing a new conserved quantum number "charm" with associated quarks, which enables them to eradicate neutral strangeness changing weak currents. A prototypal scheme is shown in Table III.

In this model (Table III) we see that $R=4 / 9+4 / 9+1 / 9+1 / 9=10 / 9$. Color can be added in a manner analogous to the usual Gell-Mann-Zweig model with the result $R=10 / 3$. This scheme is rather complicated: hadrons may be colored (i.e., not a singlet under color SU(3)) or charmed. The charmed sector brings $\mathrm{R}$ to a value of 6 . Of course the electromagnetic current has zero charm, and so no charmed particles need appear in the final 
state even if $R$ saturates the value 6 . If only color singlets are produced $R$ is reduced to $10 / 3$.

Table III Han-Nambu with Charm

\begin{tabular}{ccccc}
\hline \hline & $\mathrm{Q}$ & $\mathrm{I}_{\mathrm{z}}$ & $\mathrm{Y}$ & Charm \\
\hline $\mathrm{p}_{\mathrm{R}}^{\prime}$ & 1 & 0 & 0 & 1 \\
$\mathrm{p}_{\mathrm{R}}$ & 1 & $1 / 2$ & 1 & 0 \\
$\mathrm{n}_{\mathrm{R}}$ & 0 & $-1 / 2$ & 1 & 0 \\
$\lambda_{\mathrm{R}}$ & 0 & 0 & 0 & 0 \\
$\mathrm{p}_{\mathrm{Y}}^{\prime}$ & 1 & $1 / 2$ & -1 & 1 \\
$\mathrm{p}_{\mathrm{Y}}$ & 1 & 1 & 0 & 0 \\
$\mathrm{n}_{\mathrm{Y}}$ & 0 & 0 & 0 & 0 \\
$\lambda_{\mathrm{Y}}$ & 0 & $1 / 2$ & -1 & 0 \\
$\mathrm{p}_{\mathrm{B}}^{\prime}$ & 0 & $-1 / 2$ & -1 & 1 \\
$\mathrm{p}_{\mathrm{B}}$ & 0 & 0 & 0 & 0 \\
$\mathrm{n}_{\mathrm{B}}$ & -1 & -1 & 0 & 0 \\
$\lambda_{\mathrm{B}}$ & -1 & $-1 / 2$ & -1 & 0 \\
\hline \hline
\end{tabular}

$$
J^{e m}=J^{e m}(H a n-N a m b u)+\bar{p}_{R}^{\prime} p_{R}^{\prime}+\bar{p}_{Y}^{\prime} p_{Y}^{\prime}
$$

These schemes do not exhaust the possibilities but it is clear that experiments at CEA and SPEAR at $\mathrm{q}^{2}=25 \mathrm{GeV}^{2}$ and $80 \mathrm{GeV}^{2}$ next year (?) are of the greatest importance to quark schemes. If $\mathrm{R}$ is found to be a constant the many proposals may be narrowed down to a few or perhaps just one.

\section{WHEN SHOULD ASYMPTOPIA BE REACHED?}

Assuming that $R\left(q^{2}\right)$ does indeed approach a constant $R$ as $q^{2} \rightarrow \infty$, what sets the scale for approaching asymptopia? A first guess might be that by analogy with inelastic scattering off composite systems like atoms and nuclei, scaling should set in when $\mathrm{q}^{2}>>$ (typical level spacing of hadrons), i.e., $\mathrm{O}$ ([a few hundred $\mathrm{MeV}^{2}$ ). Indeed this is apparently observed in deep inelastic electroproduction with scaling occurring for $\left|q^{2}\right|>(1-2)$ $\mathrm{GeV}^{2}$. One should expect scaling in annihilation to set in later because the most striking singularities in $\mathrm{q}^{2}$ are timelike: the vector mesons $\rho, \omega, \phi$ 
with $\mathrm{q}^{2} \sim 1 \mathrm{GeV}^{2}$ (and $\rho^{\prime}$ with $\mathrm{q}^{2}-(2-3) \mathrm{GeV}^{2}$ ?). Presumably one needs $q^{2} \gg$ these values for scaling to occur, probably when the widths of the $1^{-}$ states become comparable with their level spacing: $q^{2}=O\left(10 \mathrm{GeV}^{2}\right)$ might seem reasonable from these arguments.

However, it can be argued threshold effects might tend to make $R\left(q^{2}\right)$ rise appreciably over a range in $\mathrm{q}^{2}$. These might either be those for producing conventional massive particles such as baryons, or unconventional particles such as colored or charmed hadrons. The baryon effect could in principle be non-negligible: ${ }^{7}$ even at low $\mathrm{q}^{2} \mathrm{e}^{+} \mathrm{e}^{-} \rightarrow \overline{\mathrm{p} p}$ is comparable with $\mathrm{e}^{+} \mathrm{e}^{-} \rightarrow \pi^{+} \pi^{-}$. Also it has been suggested 18 that the parton model may be applied to large $p_{1}$ phenomena in hadron-hadron collisions. Experiments indicate there may be relatively many baryons at large $p_{\perp}$ suggesting there may be many baryons produced at large $\mathrm{q}$ in $\mathrm{e}^{+} \mathrm{e}^{-}$annihilation. Also estimates suggest19 that asymptopia for baryon production may set in only when $\mathrm{q}^{2}>10 \mathrm{GeV}^{2}$.

As mentioned in the previous section, the asymptotic cross section expected in Han-Nambu type models increases when color octet hadron states are excited because Jem has a color octet piece. Thus $R\left(q^{2}\right)$ could have a plateau in $\mathrm{q}^{2}$ and then a rise, or else rise more gradually and continuously. Colored hadrons would presumably be rather more massive than ordinary hadrons, so asymptopia might be even later than suggested above. Detecting produced colored hadrons in $\mathrm{e}^{+} \mathrm{e}^{-}$annihilation might be very tricky: the heavier ones would decay electromagnetically and rapidly $\left(\tau<<10^{-16} \mathrm{sec}\right.$ probably). As also mentioned earlier, asymptopia could be reached in charmed theories even if charmed particles were not being produced, because the $\mathrm{Jem}$ is uncharmed. However threshold effects might make the approach rather slow. Charmed hadrons would have to decay weakly, and might have a distinctive experimental signature if they exist.

As mentioned earlier, asymptotically free gauge theories not only reproduce the standard prediction

$$
\mathrm{R}\left(\mathrm{q}^{2}\right) \equiv \frac{\sigma\left(\mathrm{e}^{+} \mathrm{e}^{-} \rightarrow \gamma \rightarrow \text { hadrons }\right)}{\sigma\left(\mathrm{e}^{+} \mathrm{e}^{-} \rightarrow \gamma \rightarrow \mu^{+} \mu^{-}\right)} \underset{\mathrm{q}^{2} \rightarrow \infty}{\longrightarrow} \mathrm{R}=\sum \mathrm{Q}_{\mathrm{i}}^{2}
$$

but also predict that scaling is approached as $1 / \ln q^{2}$ with a calculable coefficient. 6 For example in a three triplet Gell-Mann-Zweig model

$$
R\left(q^{2}\right) \underset{q^{2} \rightarrow \infty}{2} 2\left(1+\frac{4}{9} \frac{1}{\ln \left(q^{2} / Q_{0}^{2}\right)}+\cdots\right)
$$

where $Q_{0}$ is some arbitrary scale of (mass) ${ }^{2}$. The coefficient of $1 / \ln \left(q^{2} / Q_{0}^{2}\right)$ is model dependent, but always positive. Hence if the rising trend suggested by the CEA data were correct, then one would have to conclude that asymptopia is not reached for $\mathrm{q}^{2} \leq 25 \mathrm{GeV}^{2}$.

Chanowitz and Drell ${ }^{20}$ have suggested another reason why $R\left(q^{2}\right)$ may increase at present colliding beam energies, though they are agnostic about what happens to $\mathrm{R}\left(\mathrm{q}^{2}\right)$ asymptotically. Motivated largely by experimental deviations ${ }^{21}$ from a dipole form for the protons magnetic form factor at large $\mathrm{q}^{2}$, they suggest that scaling laws may gradually break down for $\left|q^{2}\right|>20 \mathrm{GeV}^{2}$. They expect a breakdown of point-like behavior of the 
constituents (partons) of hadrons corresponding to a structure generated by gluons with masses $M_{G}=10 \mathrm{GeV}$. In the region $1 \ll\left|q^{2}\right| \ll M_{G}^{2}$ they find the dipole form factor modified by a factor $\left(1-\left|q^{2}\right| / M_{G}^{2}\right)$, violation of scaling in deep inelastic electroproduction by a factor $\left(1-2\left|q^{2}\right| / M_{G}^{2}\right)$ and in $\mathrm{e}^{+} \mathrm{e}^{-} \rightarrow \gamma \rightarrow$ hadrons

$$
\sigma-\frac{1}{q^{2}}\left(1+\frac{2 q^{2}}{M_{G}^{2}}\right) \quad \text { for } 1 \ll q^{2} \ll M_{G}^{2}
$$

Chanowitz and Drell are non-committal about what happens when $q^{2} \geq M_{G}^{2}$. If the gluon coupled directly to the photon, then it would be seen as a resonance bump in the annihilation cross section. However, if it were not so coupled (e.g., if the gluon were scalar or colored), then the behavior for $q^{2}-M_{G}^{2}$ would not be predictable. In any case behavior in this model in the limit $\mathrm{q}^{2} \rightarrow \infty$ is unknown, as it faces the usual problem of a renormalizable theory with a large coupling constant.

It is amusing to consider the implications for purely leptonic processes of a large ratio $\mathrm{R}\left(\mathrm{q}^{2}\right)$. Bjorken and Frishman 22 have considered the effects of the hadronic corrections to the photon propagator in processes like $\mathrm{e}^{+} \mathrm{e}^{-} \rightarrow \mathrm{e}^{+} \mathrm{e}^{-}$and $\mathrm{e}^{+} \mathrm{e}^{-} \rightarrow \mu^{+} \mu^{-}$(see Figs. 3,4). If $\mathrm{R}\left(\mathrm{q}^{2}\right)$ were as large as



241144

FIG. 4--Hadronic vacuum polarization correction for $\mathrm{s}$ channel photon exchange in $\mathrm{e}^{+} \mathrm{e}^{-} \rightarrow \mathrm{e}^{+} \mathrm{e}^{-}$and $\mathrm{e}^{+} \mathrm{e}^{-} \rightarrow \mu^{+} \mu^{-}$. suggested by CEA and increased as a power of $\mathrm{q}^{2}$ up to the unitarity limit then the $t$-channel Born graph cross section in $\mathrm{e}^{+} \mathrm{e}^{-} \rightarrow \mathrm{e}^{+} \mathrm{e}^{-}$(see Fig. 3) could be affected by $\mathrm{O}(2 \%)$ at $-\mathrm{t} \sim 12 \mathrm{GeV}^{2}$, the maximum momentum transfer accessible at SPEAR or CEA. There could also be interesting polarization effects in $\mathrm{e}^{+} \mathrm{e}^{-} \rightarrow \mathrm{e}^{+} \mathrm{e}^{-}$because of the phase in the time-like graph shown in Fig. 4. There would of course be no corrections to the conventional lowest order QED calculation of $\mathrm{e}^{+} \mathrm{e}^{-} \rightarrow \gamma \gamma$ because it contains no internal photon propagators.

\section{QUALITATIVE FEATURES OF THE FINAL STATE}

\section{Jets or Not?}

One approach to the study of final states in $\mathrm{e}^{+} \mathrm{e}^{-}$annihilation is to build upon our experience with hadronic collisions where the final state hadrons have limited transverse momentum. If the final states in annihilation were isotropic then we might conclude that the total momentum of the final state hadrons would be similarly limited. 23 A direct consequence would be a multiplicity growing as $\mathrm{s}^{1 / 2} /\left\langle\mathrm{E}_{\pi}>\right.$ where $\left\langle\mathrm{E}_{\pi}>\sim 400 \mathrm{MeV}\right.$. Early data from CEA already give trouble for this picture, since at $q^{2} \simeq(16-25) \mathrm{GeV}^{2}$ they show $\left\langle\mathrm{n}_{\mathrm{ch}}\right\rangle-4-4.5$ suggesting $\left\langle\mathrm{n}_{\mathrm{all}}\right\rangle \sim 6-7$ and $\left\langle\mathrm{E}_{\pi}\right\rangle \sim 700 \mathrm{MeV}$.

Of course parton models do not predict isotropic final states, but jets aligned in the directions of the "produced" partons. These jets would have $\left(1+\cos ^{2} \theta\right)$ distribution if the partons had spin $-1 / 2$. Bjorken and Brodsky 23 
suggest that jets might be searched for by considering the traceless tensor

$$
T_{\alpha \beta}=\frac{\sum_{i}\left(\frac{3}{2} p_{\alpha}^{i} p_{\beta}^{i}-\frac{1}{2} \delta_{\alpha \beta}\left[p^{i}\right]^{2}\right.}{\sum_{i}\left[p^{i}\right]^{2}}
$$

where the sum over $i$ is over all particles in any particular event. The eigenvalues of $\mathrm{T}_{\alpha \beta}$ would be determined for each event. Isotropic events would give degenerate eigenvalues, while jets would give non-degenerate eigenvalues $\mathrm{a},-\mathrm{a} / 2,-\mathrm{a} / 2$, say. The diagonalization of $\mathrm{T}_{\alpha \beta}$ would determine the jet axis which should have a $\left(1+\cos ^{2} \theta\right)$ distribution.

In the parton model momenta transverse to the jet axis are limited to $\mathrm{O}(400 \mathrm{MeV})$. In this and other models ${ }^{24}$ the one particle inclusive distributions exhibit Feynman scaling. The energy conservation sum rule

$$
\sum_{\substack{\text { particle } \\ \text { types a }}} \int \frac{\mathrm{d}^{3} \mathrm{p}_{\mathrm{a}}}{\mathrm{E}_{\mathrm{a}}}\left(\frac{\mathrm{E}_{\mathrm{a}}}{\sigma_{\text {tot }}} \frac{\mathrm{d} \sigma}{\mathrm{d}^{3} \mathrm{p}_{\mathrm{a}}}\right) \mathrm{E}_{\mathrm{a}}=\sqrt{\mathrm{s}}
$$

is satisfied by having $\frac{E}{\sigma} \mathrm{d} \sigma / \mathrm{d}^{3} \mathrm{p}$ become a function of $\mathrm{x}=2|\mathrm{p}| /\left(q^{2}\right)^{1 / 2}$ as $\mathrm{q}^{2} \rightarrow \infty$.

Another possibility for the hadron distribution is that the momenta are large, so that the one particle distribution scales in $x=2|p| /\left(q^{2}\right)^{1 / 2}$, but the momenta in individual events do not have a limit transverse to any axis. This is the analogue of having $p_{\perp}-\left(Q^{2}\right)^{1 / 2}$ in the photon fragmentation region of deep inelastic scattering. 25 Early data 26 do not support this picture in electroproduction.

\section{MULTIPLICITIES AND TOPOLOGICAL CROSS SECTIONS}

Data on the total charged multiplicity are already available from CEA: more information on the multiplicity distribution and topological cross sections will soon be available from there and from SPEAR. It is commonly expected that the multiplicity will increase $\propto \ln q^{2}$, as seems to be the case in hadronic collisions. A rapidity variable $y=1 / 2 \ln (E+|p|) /(E-|p|)]$ can be introduced for particle produced in annihilation, and if the one particle inclusive rapidity distribution is flat in the central region with a height

$$
\frac{1}{\sigma} \frac{\mathrm{d} \sigma}{\mathrm{dy}}=\mathrm{C}_{\mathrm{e}^{+} \mathrm{e}^{-}}
$$

then the multiplicity is $\langle\mathrm{n}\rangle=\mathrm{C}_{\mathrm{e}^{+} \mathrm{e}^{-}} \ln \mathrm{q}^{2}+$ const. In the parton model of

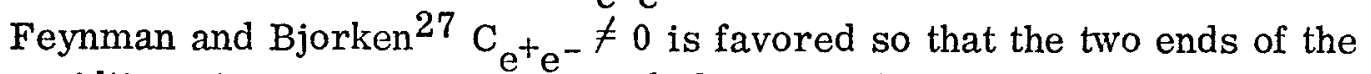
rapidity axis can communicate and eliminate their quark-like quantum numbers from the final state hadrons. In this model the multiplicities in $\mathrm{e}^{+} \mathrm{e}^{-}$ annihilation, hadron-hadron collisions and deep inelastic lepton production 
are closely related 28

$$
\begin{aligned}
& <\mathrm{n} \mathrm{e}_{\mathrm{e}^{+}}=\mathrm{C}_{\mathrm{e}^{+} \mathrm{e}^{-}} \ln \mathrm{q}^{2}+\text { const. } \\
& <\mathrm{n}>_{\text {hadrons }}=\mathrm{C}_{\mathrm{n}} \ln \mathrm{s}+\text { const. } \\
& \underset{\substack{\mathrm{ep} \\
\mathrm{n}}}{ }=\mathrm{C}_{\mathrm{e}^{+}} \mathrm{e}^{-\ln \mathrm{q}^{2}+\mathrm{C}_{\mathrm{n}} \ln (\omega-1)+\text { const. }}
\end{aligned}
$$

Preliminary data from Frascati and $\mathrm{CEA}^{2}$ indicate that $\langle\mathrm{n}\rangle \mathrm{e}^{+} \mathrm{e}^{-}$is increasing at a rate comparable with that in hadron-hadron collisions. Data on deep inelastic electroproduction also indicate that $\langle n\rangle$ increases with $\omega$ and $q^{2} .29$

One of the reasons why increasing hadronic multiplicities in annihilation are favored is that if the multiplicity were asymptotically finite, then at least one exclusive channel would have to scale:

$$
\frac{\sigma_{\text {exch }}}{\sigma_{\text {tọt }}} \underset{q^{2} \rightarrow \infty}{\longrightarrow} \text { const. }
$$

(see for example, Ref. 19). . Two-body channels like $\mathrm{e}^{+} \mathrm{e}^{-} \rightarrow \pi^{+} \pi^{-}$or $\mathrm{p} \overline{\mathrm{p}}$ seem experimentally and are expected theoretically to behave as

$$
\frac{1}{q^{2}} \quad\left[F\left(q^{2}\right)\right]^{2} \quad \text { as } q^{2} \rightarrow \infty
$$

with $F\left(q^{2}\right)$ some form factor going to zero in the limit. Quasi-two-body channels like $e^{+} e^{-} \rightarrow \rho^{+} \rho^{-}$and $\Delta^{++} \Delta^{--}$are expected to exhibit similar behavior. This is analogous to the behavior of non-diffractive exclusive channels in hadronic collisions, which are expected to fall relative to the total cross section as $\mathrm{s}^{2 \alpha-2}$ with $\alpha$ some Regge intercept $\lesssim 1 / 2$. Diffractive channels in hadronic collisions would correspond to exclusive channels which scaled so that $F\left(q^{2}\right) \rightarrow$ const as $q^{2} \rightarrow \infty$, i. e., exclusive channels with asymptotically point-like form factors. If partons were actually types of hadrons, then their production could be "diffractive" in this sense. It is easy to show


limit of the total annihilation cross-section is just the sum of the limits of the "diffractive" exclusive cross sections:

$$
\lim _{q^{2} \rightarrow \infty} q^{2} \sigma_{t o t}\left(q^{2}\right)=\sum_{n} \lim _{q^{2} \rightarrow \infty} q^{2} \sigma_{n}\left(q^{2}\right)
$$

In most parton models, partons are not actual hadrons, but there may still be "diffractive" channels. For example in the model of Landshoff and Polkinghorne, 30 the total annihilation cross section is proportional to

$$
\frac{1}{q^{2}} \int_{1}^{\mathrm{S}} \mathrm{d} \sigma \rho(\sigma) \int_{1}^{\mathrm{T}} \mathrm{d} \tau \rho(\tau)
$$


where $\rho$ is the spectral function of a parton propagator and $\mathrm{S}$ and $\mathrm{T}$ are upper bounds of order $\mathrm{q}^{2}$. There is a normalization condition

$$
\int_{1}^{\infty} \mathrm{d} \sigma \rho(\sigma)=\text { finite }
$$

In this model, one could truncate the $\sigma$ and $\tau$ integrals at any finite values $\mathrm{S}_{0}$ and $\mathrm{T}_{0}$, and still get a contribution to the cross section which goes as $1 / q^{2}$. If there is a mechanism adjusting the quantum numbers in the final state so that only normal hadrons appear, and if this does not substantially change the rapidity distribution in individual events, this would correspond to the production of two finite mass jets of hadrons widely separated in rapidity. Since there are only a finite number of hadronic states with masses less than any given value, this means that at least some quasi-two-body exclusive channels should be point-like or "diffractive". Partons appearing as hadrons would correspond to $\delta$-function contributions to $\rho(\sigma)$. However, this may involve taking the details of the final state in the Landshoff-Polkinghorne model too seriously: perhaps one should only expect some of the predictions for inclusive distributions to be valid.

In the parton model of Bjorken, Feynman and others ${ }^{27,28}$ all exclusive channels in annihilation are expected to fall relative to the total cross section, and they all conspire in a "multiperipheral" manner to build up the total cross section. Naively in the Landshoff-Polkinghorne 30 the total cross section, even if the multiplicity increases logarithmically, is completely constructed out of point-like exclusive channels asymptotically, as in diffractive excitation 31 models of hadron collisions. How can we discriminate between these two pictures? One way is to look directly for the scaling exclusive channels. Another is to look at the topological cross sections and see whether they have the "multiperipheral" structure of Fig. 5, or the "diffractive" structure of Fig. 6, or some combination of the two.

Light-cone analysis allows a logarithmically increasing multiplicity. 32 If the bilocal operator is expressible as a finite sum of factorizable terms

$$
\mathrm{B}(\mathrm{x}, 0) \sim \sum \phi_{\mathbf{i}}(\mathrm{x}) \phi_{\mathrm{i}}^{\prime}(0)
$$

then the final state structure must be similar to the Landshoff-Polkinghorne model. 33 However, a more general structure for the bilocal, as found for example in canonical manipulations of the quark-vector gluon model, does

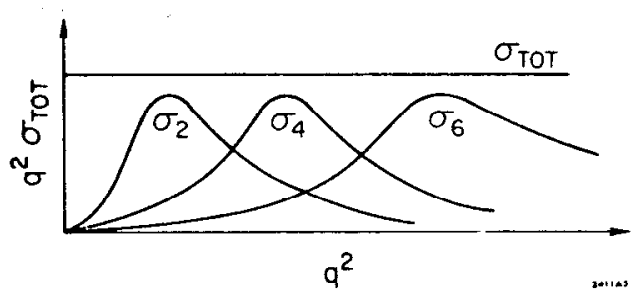

FIG. 5--Schematic representation of "multiperipheral-like" partial cross sections adding up to a scaling cross section.

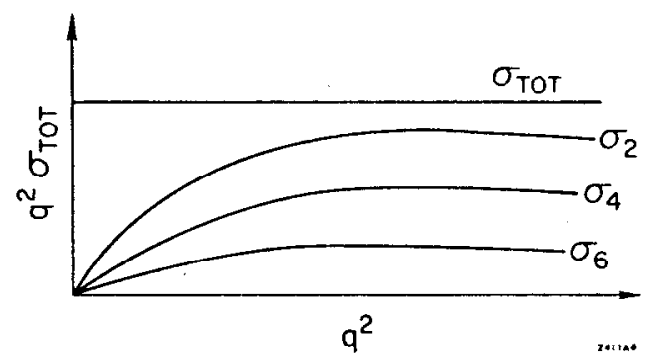

FIG. 6--Schematic representation of "diffractive-like" partial cross sections adding up to a scaling cross section. 
not imply any two jet structure. In the Landshoff-Polkinghorne model with logarithmic multiplicity scaling in annihilation is approached from below, and no more rapidly than $\left(q^{2}\right)^{-1 / 2} .34$

These various different pictures say different things about moments of the multiplicity distribution. In the Landshoff-Polkinghorne model with logarithmic multiplicity, $f_{2}$ and the hi her moments grow like powers of $q^{2}$. Naively one might expect $f_{2}-\left(q^{2}\right)^{1 / 2}, 35$ as in the simple formulation of the diffractive excitation model. But just as $t_{\text {min }}$ effects can cut down $f_{2}$ in the diffractive excitation model, $35 \mathrm{f}_{2}$ in annihilation could increase more slowly. For example

$$
\sigma_{n}-\frac{\theta\left(\left(q^{2}\right)^{\epsilon}-n\right)}{n^{2}} \quad 0<\epsilon<1 / 2
$$

implies

$$
\langle\mathrm{n}\rangle=\sum_{\mathrm{n}=1}^{\left(\mathrm{q}^{2}\right)^{\epsilon}} \frac{1}{\mathrm{n}}-\epsilon \ln \mathrm{q}^{2}
$$

and

$$
f_{2}-\left\langle n^{2}>-\left(q^{2}\right)^{\epsilon}\right.
$$

In the conventional "multiperipheral" parton model of Bjorken and Feynman, 28,29 all the multiplicity moments are expected to grow as powers of $\ln q^{2}$. Unfortunately experiments at CEA and SPEAR will presumably be at values of $\mathrm{q}^{2}$ too low to discriminate between a logarithm and a small power of $\mathrm{q}^{2}$.

\section{ACKNOWLEDGMENTS}

We thank J. D. Bjorken, M. S. Chanowitz, E. W. Colglazier, Y. Frishman, R. L. Jaffe, H. R. Quinn and J. Stack for useful conver sations. One of us(J.E.) thanks SLAC for its generous hospitality while this talk was prepared. 


\section{REFERENCES}

1. J. D. Bjorken, Phys. Rev. 148, 1467 (1966).

V. N. Gribov, B. L. Ioffe and I. Pomeranchuk, Yadern. Fiz. 6, 586 (1967).

2. A. Litke, et al., Phys. Rev. Letters 30,1189 (1973) and talk presented by H. Newman at the SLAC Summer Institute in Particle Physics (1973).

3. N. Cabibbo, G. Parisi and M. Testa, Letterc al Nuovo Cimento, IV, 35 (1970).

4. K. G. Wilson, Phys. Rev. 179, 1499 (1969).

5. D. J. Gross and F. Wilczek, Phys. Rev, Letters 30, 1343 (1973)。

H. D. Politzer, Phys. Rev. Letters 30, 1346 (1973)。 It appears this result was also known to G. $t^{\prime}$ Hooft.

6. T. Appelquist and H. Gcorgi, Harvard preprint (1973). A. Zee, "Electron-Positron Annihilation in Stagnant Field Theories," Rockefeller Univ. preprint (1973).

7. R. L. Kingsley, Nucl. Phys. B 46, 615 (1972). P. M. Fishbane and

R. L. Kingsley, Preprint IT P-SB-73-33 (1973).

8. S. Weinberg, Phys. Rev. Letters 19,1264 (1967). A. Salam and J. C. Ward, Nuovo Cimento 11, $568(1959)$. H. Georgi and S. L. Glashow, Phys. Rev. Letters 28, 1494 (1972).

9. H. J. Lipkin, Weizman institute preprint (1973).

10. D. J. Gross and F. Wilczek, preprint NAL THY 73-49 (1973) .

11. M. Gcll-Mann, Phys. Letters 8,214 (1964). G. Zweig, CERN reports TH -401 and TH-412, unpublished (1964).

12. K. G. Wilson, op. cit. R. J. Crewther, Phys. Rev. Letters 28, 1421 (1972).

13. H. Fritzsch and M. Gell-Mann, Proceedings of the XVI International Conference on High Energy Physics. J. D. Jackson and A. Roberts, cd. Vol. 2, p. 135 (1972) and references therein.

14. Y. Nambu, in "Preludes in Theoretical Physics," A. de-Shalit, H. Feshbach, L. Van Hove, ed. (North Holland, Amsterdam, 1966) p. 133.

15. M. Y. Han and Y. Nambu, Phys. Rev. 139, B 1006 (1965).

16. S. L. Glashow, J. Iliopoulos and L. Maiani, Phys. Rev. D 2,1285 (1970).

17. J. D. Bjorken, private communication.

18. S. Berman, J. D. Bjorken and J. Kogut, Phys. Rev. D $\underline{4}, 3388$ (1971).

19. J. D. Bjorken and J. Kogut, Phys. Rev. D $\underline{8}, 1341$ (1973).

20. M. Chanowitz and S. D. Drell, Phys. Rev. Letters 30, 807 (1973).

21. D. Coward, et al., Phys. Rev. Letters $20,292(1968)$. T. Massam and A. Zichichi, Nuovo Cimento Letters 1 , 387 (1969).

22. J. D. Bjorken and Y. Frishman, private communication.

23. J. D. Bjorken and S. J. Brodsky, Phys. Rev. D 1, 1416 (1970) .

24. S. D. Drell, D. J. Levy and T. -M. Yan, Phys. Rev. D 1,1617 (1970). J. Ellis, Phys. Letters 35 B, 537 (1971).

25. J. Stack, Phys. Rev. Letters 28, 57 (1972). M. Gonzalez and J. Weis, Univ. of Washington preprint, 1973.

26. E. Lazarus, et al., Phys. Rev. Letters 29, 743 (1972). C. J. Bebek, et al., Phys. Rev. Letters 30, 624 (1973).

27. $\bar{R}, \bar{P}$. Feynman in "Photon $\vec{H}$ adron Interactions"(W. A. Benjamin, 1972). J. D. Bjorken, "Proceedings of the Fifth International Symposium on Electron and Photon Interactions at High Energies", at Cornell Univ., 
1971, N. B. Mistry, ed. and Phys. Rev. D $\underline{7}, 282$ (1973). S. Berman

J. D. Bjorken and J. Kogut, op.cit.

28. R. Cahn, J. Cleymans and E. W. Colglazier, Phys. Letters $\underline{43}$ B, 323 (1973).

29. K. Berkelman, et al., CLNS-240 (1973) preprint.

30. R. L. Kingsley, P. V. Landshoff, C. Nash and J. C. Polkinghorne, preprint DAMTP 73/19 (1973)。

31. R. Hwa, Phys. Rev. Letters 26, 1143 (1971).

32. J. Ellis and Y. Frishman, Phys. Rev. Letters 31, 135 (1973).

33. J. Stack, Univ. of Illinois preprint, ILL-(TH)-73-4 (1973).

34. H. Osborn and G. Woo, preprint DAMTP, 73/13, April 1973.

35. D. M. Scott, preprint DAMTP 73/26 (1973).

36. R. Hwa, Phys. Rev. Letters 28, 1487 (1972). 